\title{
Design of the Stenting and Aggressive Medical Management for Preventing Recurrent Stroke in Intracranial Stenosis Trial
}

\author{
Marc I. Chimowitz, MBChB' ${ }^{1}$, Michael J. Lynn, MS², Tanya N. Turan, MD¹, David Fiorella, \\ MD, $\mathrm{PhD}^{3}$, Bethany F. Lane, RN${ }^{2}$, Scott Janis, $\mathrm{PhD}^{4}$, and Colin P. Derdeyn, $\mathrm{MD}^{5}$ for the \\ SAMMPRIS Investigators \\ ${ }^{1}$ Department of Neurosciences, Stroke Center, Medical University of South Carolina, Charleston, \\ SC \\ ${ }^{2}$ Department of Biostatistics, Emory University Rollins School of Public Health, Atlanta, GA \\ ${ }^{3}$ Department of Neurosurgery, Cerebrovascular Center, State University of New York, Stony \\ Brook, NY \\ ${ }^{4}$ National Institute of Neurological Disorders and Stroke, National Institute of Health, Bethesda, \\ MD \\ ${ }^{5}$ Mallinckdrodt Institute of Radiology and the Departments of Neurology and Neurosurgery; \\ Washington University School of Medicine, St Louis MO
}

\begin{abstract}
Background-Patients with recent transient ischemic attack or stroke caused by 70-99\% stenosis of a major intracranial artery are at high risk of recurrent stroke on usual medical management, suggesting the need for alternative therapies for this disease.
\end{abstract}

\footnotetext{
(C) 2011 National Stroke Association. Published by Elsevier Inc. All rights reserved.

Corresponding author: Marc I. Chimowitz, MBChB, MUSC Stroke Program, 19 Hagood Ave. Harborview Office Tower, Suite 501, Charleston, SC 29425, phone: 843-792-3020, fax: 843-792-2484, mchimow@ musc.edu.

Publisher's Disclaimer: This is a PDF file of an unedited manuscript that has been accepted for publication. As a service to our customers we are providing this early version of the manuscript. The manuscript will undergo copyediting, typesetting, and review of the resulting proof before it is published in its final citable form. Please note that during the production process errors may be discovered which could affect the content, and all legal disclaimers that apply to the journal pertain.

Disclosures

All the authors serve on the Executive Committee of the Stenting and Aggressive Medical Management for Preventing Recurrent stroke in Intracranial Stenosis (SAMMPRIS) trial which is funded by the National Institute of Neurological Disorders and Stroke (grant number: U01 NS058728). All but one (SJ) receive salary support from the SAMMPRIS grant. Some of these authors receive additional support as listed below.

Marc Chimowitz, MBChB is the grant recipient (U01 NS058728) for the NINDS funded clinical trial described in this paper. He has also received research grants from NINDS to fund the WASID trial (1 R01 NS36643) and to fund other research on intracranial stenosis (1 K24 NS050307 and 1 R01 NS051688). He currently serves on the stroke adjudication committee of an industry funded osteoporosis drug trial (Merck and Co., Inc.) and on the DSMB of another industry funded patent foramen ovale closure trial (W.L Gore and Associates) and is compensated for those activities.

Tanya N. Turan, MD is a past recipient of funding from the American Academy of Neurology (AAN) Foundation Clinical Research Training Fellowship and is the current recipient of a K23 grant from NIH/NINDS (1 K23 NS069668-01A1)

Michael J. Lynn, MS receives grant support from the National Eye Institute. He is the principal investigator of the Coordinating Center for Infant Aphakia Treatment Study (EY013287) and a co-investigator on the Core Grant for Vision Research (EY006360). David Fiorella MD, PhD has received institutional research support from Seimens Medical and Microvention, consulting fees from Micrus - Johnson and Johnson, EV3 / Covidian and Microvention, and royalties from Micrus - Johnson and Johnson.

Scott Janis PhD is a program director at the National Institute of Neurological Disorders and Stroke

Colin Derdeyn MD receives other grant support from the NINDS (P50 55977; R01 NS051631). He is also on the Scientific Advisory Board for W.L Gore and Associates and is the Chair of the Scientific Advisory Board for Pulse Therapeutics.
} 
Methods-The Stenting and Aggressive Medical Management for Preventing Recurrent stroke in Intracranial Stenosis trial is an ongoing, randomized, multicenter, two-arm trial that will determine whether intracranial angioplasty and stenting adds benefit to aggressive medical management alone for preventing the primary endpoint (any stroke or death within 30 days after enrollment or after any revascularization procedure of the qualifying lesion during follow-up, or stroke in the territory of the symptomatic intracranial artery beyond 30 days) during a mean follow-up of 2 years in patients with recent TIA or stroke caused by 70-99\% stenosis of a major intracranial artery. Aggressive medical management in both arms consists of aspirin $325 \mathrm{mg}$ per day, clopidogrel $75 \mathrm{mg}$ per day for 90 days after enrollment, intensive risk factor management primarily targeting systolic blood pressure $<140 \mathrm{~mm} \mathrm{Hg}(<130 \mathrm{~mm} \mathrm{Hg}$ in diabetics) and low density cholesterol $<70 \mathrm{mg} / \mathrm{dl}$, and a lifetsyle modification program. The sample size required todetect a 35\% reduction in the rate of the primary endpoint from angioplasty and stenting based on the log-rank test with an alpha of $0.05,80 \%$ power, and adjusting for a $2 \%$ loss to follow-up and $5 \%$ crossover from the medical to the stenting arm is 382 patients per group.

Conclusion-This is the first randomized trial to compare intracranial angioplasty and stenting with medical therapy and to incorporate intensive management of multiple risk factors and a lifestyle modification program in the study design. Hopefully, the results of the trial will lead to more effective therapy for this high-risk disease.

\section{Introduction}

Intracranial arterial atherosclerotic stenosis is an important cause of ischemic stroke, especially in Blacks, Asians, and Hispanics (1-5). Patients with recent transient ischemic attack (TIA) or stroke and severe stenosis (70\%-99\%) are at particularly high risk of recurrent stroke (23\% at 1 year) despite the use of aspirin and usual management of risk factors $(6,7)$. As such, this patient cohort stands to gain the most from alternative therapies. The Stenting and Aggressive Medical Management for Preventing Recurrent Stroke in Intracranial Stenosis (SAMMPRIS) Trial is the first randomized trial designed to compare percutaneous transluminal angioplasty and stenting (PTAS) plus aggressive medical management with aggressive medical management alone in high-risk patients with intracranial stenosis (8). This paper describes the design of the trial, the rationale for key components of the design, and summarizes the unique features of the design.

\section{Methods}

\section{Overall Design}

SAMMPRIS is an on-going, investigator-initiated, randomized, clinical trial funded by the National Institute of Neurological Disorders and Stroke (NINDS) that is being conducted at 50 sites in the United States. The primary aim is to determine whether PTAS (using the Gateway® PTA Balloon Catheter and Wingspan ${ }^{\circledR}$ Stent System, both manufactured by Boston Scientific Corporation) combined with aggressive medical management is superior to aggressive medical management alone for preventing the primary endpoint (any stroke or death within 30 days after enrollment or after any revascularization procedure of the qualifying lesion during follow-up, or stroke in the territory of the symptomatic artery beyond 30 days) in high-risk patients with intracranial stenosis (see criterion \# 1 in table 1 for definition of high-risk). The study protocol was approved by each site's Institutional Review Board and the Data Safety Management Board (DSMB) appointed by NINDS. The Food and Drug Administration (FDA) issued an Investigational Device Exemption (G050157) to carry out the study. 


\section{Credentialing of Interventionists}

Interventionists interested in participating in the trial were required to submit procedure notes and discharge or follow up notes from their most recent 20 consecutive intracranial stent or angioplasty cases. Only cases performed as the primary operator were accepted and procedures performed during fellowship or as an assistant were not accepted. Follow up clinic notes or discharge summaries were required for all cases to document outcome. Accepted cases, in order of preference, were procedures using 1) the Wingspan stent for intracranial atherosclerosis; 2) a balloon-mounted coronary stent for intracranial atherosclerosis; 3 ) a self-expanding stent for aneurysm (owing to the similarity in the delivery device to Wingspan); and finally 4) angioplasty alone for intracranial atherosclerosis if the interventionist did not have 20 stenting cases. A minimum of three Wingspan cases was required to be considered for credentialing. The clinical presentation, procedural details, device used, lesion location, and outcome for each of the 20 cases were abstracted to a spreadsheet. The spreadsheet and the supporting documents were reviewed by the Credentialing Committee consisting of seven experienced, senior interventionists. Cases with procedural or post-procedural complications were flagged for closer review and discussion. Submissions were not reviewed until all case data were complete. The committee's charge was to determine if the interventionist demonstrated sufficient experience, judgement and skill to participate in the trial. The results of the committee deliberations were approval or non-approval, and approval required a unanimous vote. If the only concern was insufficient experience with the Wingspan device, the recommendation was to reject the application but reconsider after a requisite number of additional cases were performed.

\section{Patient Population}

The population of interest in this trial includes both in-patients and out-patients at the participating sites who have TIA or non-disabling ischemic stroke (modified Rankin score $\leq$ 3 ) and intracranial stenosis. The diagnostic evaluation for intracranial stenosis at each site is at the discretion of the local investigator and can include transcranial Doppler (TCD) $(9,10)$, magnetic resonance angiography (MRA) $(10,11)$, or computerized tomographic angiography (CTA) (12), but catheter angiography is ultimately required to confirm 70-99\% stenosis by the WASID criteria (13) for a patient to qualify. Patients are enrolled on the basis of angiogram readings performed on-site but all angiograms are sent to the coordinating center for central reading by the same neuroradiologist who read the angiograms in the WASID trial. Early in the trial, a high percentage of angiograms were read as $270 \%$ by site readers but $<70 \%$ by the central reader so we instituted a policy requiring that jpeg images of all subsequent angiograms read locally as $70-79 \%$ stenosis be sent by email and one of the interventional principal investigators had to confirm that the stenosis was at least $70 \%$ in order for the patient to qualify. The inclusion and exclusion criteria for the trial are provided in tables 1 and 2. Patients give written informed consent to participate and those who do not undergo angiography as part of routine care give consent for angiography as part of the study protocol.

\section{Randomization and Treatments}

Patients are randomized (1:1) to aggressive medical management alone or to PTAS plus aggressive medical management. The sequence of treatment assignments are stratified by center and produced at the Statistical Coordinating Center using a pseudo-random number generator with randomly permuted block sizes.

Aggressive medical management is identical in both treatment arms and consists of enteric coated aspirin $325 \mathrm{mg}$ per day for the entire follow-up, clopidogrel $75 \mathrm{mg}$ per day for 90 days after enrollment, intensive management of the primary risk factors (systolic blood 
pressure and low density lipoprotein (LDL)), as well as management of secondary risk factors (diabetes, non-high density lipoprotein (non-HDL), smoking, weight, exercise). The study provides aspirin and clopidogrel, rosuvastatin, one medication from each major class of antihypertensive agents (diuretic, angiotensin converting enzyme inhibitor, angiotensin receptor blocker, beta-blocker, long-acting calcium channel antagonist, potassium-sparing diuretic, vasodilator, central alpha agonist), and the lifestyle modification program to study patients. All study medications except rosuvastatin are provided through retail pharmacies near where the patients live. The rosuvastatin is shipped to sites for distribution to the patients by the study coordinators.

Patients randomized to PTAS are required to undergo PTAS within three business days of randomization. Patients who have not been on clopidogrel $75 \mathrm{mg}$ per day for five days prior to PTAS are given a $600 \mathrm{mg}$ loading dose of clopidogrel between 6 and 24 hours before the PTAS procedure. The study protocol requires that the PTAS procedure is performed under general anesthesia by a SAMMPRIS-credentialed interventionist who must be the primary operator. The procedure is typically performed via a trans-femoral placed $6 \mathrm{~F}$ long sheath or guiding catheter but a radial or brachial artery approach is permitted if that is the operator's preference. The stenotic lesion is primarily crossed with a standard 0.014 "microcathetermicrowire system under high magnification fluoroscopic roadmap control. Once across the lesion, the microcatheter is exchanged over a $300 \mathrm{~cm}, 0.014$ " microwire for a Gateway angioplasty balloon. Following angioplasty, the balloon catheter is exchanged for a Wingspan stent delivery system and the self-expanding Wingspan stent is deployed across the stenosis. If the residual stenosis after inserting the Wingspan stent is $\geq 50 \%$, the study protocol allows for post-dilation with a new balloon catheter. The PTAS protocol requires that intravenous heparin is given to maintain the activated clotting time (ACT) between 250-300 seconds throughout the procedure. IIb / IIIa agents can only be used if an intraluminal thrombosis develops during the procedure. If so, the targeted ACT is to be lowered to less than 220 seconds to minimize the risk of bleeding. The protocol requires frequent measurements of blood pressure during the procedure and at least every two hours over the next 24 hours while the patient is monitored in an intensive care or step-down unit. If the blood pressure is greater than $150 \mathrm{~mm} \mathrm{Hg}$ systolic or $95 \mathrm{~mm} \mathrm{Hg}$ diastolic, intravenous antihypertensive treatment is required to lower the risk of reperfusion injury once PTAS has been performed (14).

\section{Follow-Up Schedule}

The site neurologist and the coordinator are required to evaluate study patients at enrollment. To ensure that all adverse events that occur in the peri-procedural period (in the PTAS arm) and in the equivalent peri-enrollment period (in the medical arm) are captured, the study coordinator contacts the patient (by telephone or in person if patient still hospitalized) 4 days after enrollment. If a potential adverse event has occurred, the neurologist evaluates the patient as soon as possible. Follow-up visits continue to occur at 30 days, 4 months, and then every 4 months until the first of the following: 90 days after a primary endpoint, death, 3 years of follow-up, or the close-out visit for the trial that will occur when the last patient enrolled in the trial has been followed for 1 year. At each followup visit, the patient's medications are reviewed, the patient is weighed, and blood pressure readings are taken in a standard way using the study-supplied Omron HEM-705CP automated blood pressure device (Omron Healthcare, Inc., Vernon Hills, Illinois). Additionally, the patient is interviewed and examined by the site neurologist to determine if any adverse events have occurred, risk factors are managed as described below, rosuvastatin is dispensed by the coordinator, prescriptions for the other study medications are provided (or called in to the pharmacy), and the Modified Rankin, Barthel Index, NIH Stroke Scale, 
and the Montreal Cognitive Assessment evaluations are performed (latter assessed at study entry, 4 months, 12 months and close-out visit).

Compliance with antithrombotic therapy is checked by counting study-supplied aspirin tablets at each follow-up visit. Additionally, the coordinator calls the patient by phone 90 days after enrollment to obtain verbal pill counts of the aspirin and clopidogrel and, if there are any clopidogrel tablets left, the patient is instructed to stop taking them. Patients are permitted to take non-study provided clopidogrel beyond 90 days only if a physician not involved in the trial recommends it for a non-neurologic indication (e.g. coronary or peripheral vascular disease).

\section{Risk Factor Management}

Risk factor management at each site is managed by a team including the site neurologist and study coordinator, and a lifestyle coach (from Nationwide Better Health - INTERVENT, Columbus, $\mathrm{OH})$. The neurologist and coordinator follow the study algorithms for managing the primary risk factors, targeting systolic blood pressure $<140 \mathrm{~mm} \mathrm{Hg}(<130 \mathrm{~mm} \mathrm{Hg}$ if diabetic) and LDL $<70 \mathrm{mg} / \mathrm{dl}$. At each follow-up visit, if a patient's systolic blood pressure is above target, an adjustment in the antihypertensive regimen is made and the patient returns for a blood pressure check in 30 days. Once the reading is within target, the patient resumes the regular follow-up schedule of every 4 months. Adjustments of the statin dose to achieve an LDL < $70 \mathrm{mg}$ are based on the patient's blood samples sent to the Emory Lipid Laboratory (Atlanta, GA) for measurement of direct LDL at study entry, 30 days and 4 months after enrollment. Beyond 4 months, adjustments are based on site LDL levels.

The site neurologists and coordinators are also responsible for overseeing the management of secondary risk factors. In diabetics, the protocol recommends that hemoglobin A1c be checked at enrollment and at least twice a year (quarterly if the patient is not meeting glycemic goals or if diabetic medications are changed) targeting a level of $<7 \%$ (15). The study neurologist works with the diabetic patient's primary care physician and endocrinologist (if needed) to achieve good diabetic control. Non-HDL cholesterol $<100$ $\mathrm{mg} / \mathrm{dL}$ is also an important secondary target (16). Once the LDL is $<70 \mathrm{mg} / \mathrm{dl}$ or LDL lowering has been maximized, if non-HDL cholesterol is $\geq 100 \mathrm{mg} / \mathrm{dl}$ and triglycerides are $\geq 200 \mathrm{mg} / \mathrm{dl}$, fenofibrate $145 \mathrm{mg}$ per day is recommended. The targets for other secondary risk factors are smoking cessation (17), body mass index (BMI) $<25 \mathrm{~kg} / \mathrm{m}^{2}$ if the enrollment BMI is $25-27 \mathrm{~kg} / \mathrm{m}^{2}$ or $10 \%$ weight loss if the enrollment BMI is $>27 \mathrm{~kg} / \mathrm{m}^{2}(18)$, and moderate intensity exercise at least 3 times per week for 30 minutes per session in patients able to participate. To help set and achieve goals for lifestyle changes that assist with risk factor management, the lifestyle coach performs an initial assessment of the patient's current health status, readiness for change, and eating, exercise, tobacco and other lifestyle habits in order to develop the patient's personal action plan, with recommendations for achieving realistic short and long-term goals. This initial assessment is done within 2 weeks of enrollment. Subsequently, the coach calls the patient every 2 weeks for the first 3 months and then every 4 weeks throughout follow-up to provide on-going lifestyle health coaching, support, motivation, re-evaluation and revision of the patient's personal action plan. These calls typically last 15-20 minutes. The goals and action plan reports, which are mailed to the patient, are also available to the patient on-line and to the study investigators through the study's electronic data capture system.

Oversight of risk factor management at each site is performed by the Director of Risk Factor Management at the Clinical Coordinating Center. With assistance from experts in hypertension, lipid disorders, and diabetes on the Risk Factor Advisory Committee, the Risk Factor Director also provides recommendations to site investigators on individual study patients with difficult to control risk factors. 


\section{Primary and Secondary Endpoints}

Primary endpoints are 1) any stroke or death within 30 days after enrollment, 2) any stroke or death within 30 days after a revascularization procedure of the qualifying lesion during follow-up, or 3) ischemic stroke in the territory of the qualifying artery beyond 30 days. The rationale for including 1 and 2 as primary endpoints is to capture these serious potential complications of PTAS and general anesthesia. Ischemic stroke is defined as a new focal neurological deficit of sudden onset, lasting at least 24 hours that is not associated with a hemorrhage on brain CT or MRI. Ischemic strokes are classified as in or out of the territory of the symptomatic intracranial artery. Symptomatic brain hemorrhage is defined as parenchymal, subarachnoid, or intraventricular hemorrhage detected by CT or MRI that is associated with new neurological signs or symptoms (headache, change in level of consciousness, focal neurological symptoms) lasting $\geq 24$ hours or a seizure, and is only a primary endpoint if it occurs with 30 days after enrollment or within 30 days after a revascularization procedure of the qualifying lesion during follow-up.

Secondary endpoints include 1) disabling stroke, 2) any stroke or death, 3) myocardial infarction, 4) major non-stroke hemorrhage (i.e., major systemic hemorrhage, subdural hemorrhage, epidural hemorrhage), 5) functional outcome at the end of follow-up measured by the Rankin Scale and Barthel Index, 6) cognitive outcome at end of follow-up measured by the Montreal Cognitive Assessment (MoCA). Major non-stroke hemorrhage is defined as any subdural or epidural hemorrhage or a systemic hemorrhage requiring any of the following: hospitalization, blood transfusion, or surgery.

\section{Assessment of Adverse Events}

If a potential endpoint occurs, patients are examined by the study neurologist as soon as possible and preferably within 24 hours of the event. If a stroke is suspected, MRI or CT of the brain is done. Given that the treatment assignment is known to the principal site neurologist, a second site neurologist, who is unaware of treatment assignment, is also required to evaluate a patient with an event that may be difficult to classify as a stroke, such as a prolonged TIA (lasting > 1 hour) or mild stroke (an increase in the patient's NIH Stroke Scale of $\leq 4$ from study entry). Both the blinded and unblinded neurologists' assessments are sent for central adjudication. All potential endpoints are adjudicated by independent panels of neurologists and cardiologists who are unaware of treatment assignments.

\section{Data Management and Imaging Center}

Data management is the responsibility of the Statistical Coordinating Center. Study data are entered electronically by the site coordinators into a central server running a commercial software system called DataFax (www.datafax.com). A variety of secure web applications are used for trial management including entry, tracking, and storage of all regulatory documents. CDs of all brain imaging (MRI and CT) and diagnostic angiography done for the qualifying event, the PTAS procedure angiograms, and any brain imaging or vascular imaging (MRA, CTA, angiography) done during follow-up are sent to the Imaging Center (at Washington University) where the images are uploaded to a web-based imaging database that allows the central angiogram and brain imaging readers to read the brain and vascular imaging remotely.

\section{Site Monitoring Visits}

Prior to beginning enrollment, all investigators attended a training conference. The SAMMPRIS Project Manager visited each site for an initial monitoring visit after the first 1-2 patients at the site completed the initial 30 day follow-up visit. All sites subsequently have a minimum of two monitoring visits per year (more if problems are identified). The 
follow-up monitoring is done by clinical monitors from the Regulatory and Clinical Research Institute (RCRI) clinical research organization (Minneapolis, MN). Source document verification is done for all case report forms in the electronic database. Patient medical records are reviewed to verify that enrollment criteria were met, protocol requirements are being followed, and adverse event reporting is accurate.

\section{Statistical Considerations}

The expected mean duration of follow-up is two years (range $1-3$ years). The sample size required to have $80 \%$ power to detect a $35 \%$ relative risk reduction in the primary endpoint (estimated rate $24.7 \%$ at two years in the medical arm vs. $16.1 \%$ in the PTAS arm) using a two-sided log-rank test with probability of a Type I error $=0.05$, a $2 \%$ lost to follow-up rate, and a 5\% crossover from the medical to the PTAS arm is 382 patients per group. The primary endpoint rate for the medical therapy alone group was based on analysis of WASID patients with the SAMMPRIS high-risk criteria (i.e., 70-99\% stenosis and TIA or minor stroke within 30 days prior to enrollment) and assumed a $15 \%$ relative risk reduction from aggressive medical management. The 35\% relative risk reduction from PTAS was based largely on the results of a national survey sent to stroke neurologists and interventionists to determine the minimal benefit from PTAS that would make it the treatment of choice of a majority of respondents if the primary endpoint rate was $25 \%$ at 2 years in the medical arm (19). The data from two Wingspan registries suggested that a $35 \%$ relative risk reduction was achievable $(20,21)$. The primary hypothesis will be tested using a two-sided log-rank test to compare the time to primary endpoint between the treatment groups. Patients lost to follow-up will be censored at the last contact date. A hazard ratio (medical arm / PTAS arm) will be calculated using Cox proportional hazards regression. All analyses will be intentionto treat unless specified otherwise. Pre-planned secondary analyses include identifying subgroups most versus least likely to benefit from PTAS, comparing outcomes in patients who achieve versus don't achieve risk factor goals, and comparing outcomes of patients treated with aggressive medical therapy alone in SAMMPRIS versus similar historical controls in WASID (i.e. those with the SAMMPRIS entrance criteria).

\section{Data Safety Monitoring Board (DSMB)}

The DSMB meets every 6 months to review the study progress and accumulated data. The main responsibilities of the DSMB are to ensure that study participants are not exposed to unnecessary or unreasonable risks and that the trial is conducted with high scientific and ethical standards. The DSMB is assisted by two safety monitors (one medical, one interventional) who review data on individual cases with adverse events. Two interim efficacy analyses are planned for when approximately $33 \%$ and $66 \%$ of the required primary endpoints have occurred (22).

\section{Monitoring the Performance of PTAS in the Trial}

To ensure patient safety and adherence to the PTAS protocol, the procedure notes from all PTAS procedures are reviewed. PTAS procedure data is first reviewed by the clinical research nurse at the Statistical Center. If an adverse event is identified, data related to the event (including the interventional procedure note) is promptly referred to the interventional safety monitor for review. Any concerns regarding procedural technique or protocol compliance are relayed to the DSMB and the interventional co-principal investigators for investigation and potential action. All procedure notes from cases not referred to the interventional safety monitor are reviewed by one of the two interventional co-principal investigators. Interventionists are contacted by the co-principal investigators if there are any questions raised regarding technique or protocol adherence. In addition to the case-by case review, thresholds for automatic flagging of interventionists and sites are in place that result in investigation of any single occurrence of vessel rupture or if any two of the first 10 cases 
at a site result in procedural-related serious adverse events or technical difficulties. Examples of events that result in an investigation are if an operator fails to deploy the stent in any two of the first 10 cases or if an ischemic stroke occurred in case 2 and a distal embolus occurred in case 10 at a site. Outcomes of these investigations can range from no action to a recommendation to suspend or terminate the interventionist or the site by consensus opinion of the two co-principal investigators. Decisions to suspend or terminate an interventionist or site must be presented to the Credentialing Committee for approval.

\section{Rationale for Key Elements of Study Design}

Why were patients with remote symptoms and $50-69 \%$ stenosis excluded from SAMMPRIS?-WASID showed that severity of stenosis was the most powerful predictor for recurrent stroke in the territory of the the stenotic artery. The cumulative rates of stroke in the territory in patients with $270 \%$ stenosis were $18 \%$ at one year and $19 \%$ at two years, whereas the rates of stroke in the territory in patients with $<70 \%$ stenosis were $6 \%$ at 1 year and $10 \%$ at 2 years (7). Time from qualifying event to enrollment was also a powerful predictor of recurrent stroke, especially in patients with $70 \%-99 \%$ stenosis: in 132 patients with $70 \%-99 \%$ stenosis who had their qualifying events within 30 days prior to enrollment, the 1 and 2-year rates of stroke in the territory were $22.9 \%$ and $25 \%$, respectively, whereas in 71 patients with $70 \%-99 \%$ stenosis and a qualifying event more than 30 days prior to enrollment, the 1 and 2-year rates of stroke in the territory were both 9\% (unpublished data from WASID, Michael Lynn, Emory University, 2007). Given the inherent peri-procedural risk of PTAS (30-day rate of stroke and death of $4.5-9.6 \%$ $(20,21,23,24))$ and the relatively low risk of stroke in patients with remote symptoms or < $70 \%$ stenosis, including these patients in SAMMPRIS would have required a much larger sample size. Studying these patients in a subsequent trial may be worth considering if SAMMPRIS shows a benefit from PTAS in high-risk patients.

Why was the Wingspan stent chosen to be studied in SAMMPRIS?-Wingspan is the only FDA approved stent for atherosclerotic intracranial stenosis and has been available for clinical use since August 3, 2005 under an HDE (humanitarian device exemption) for patients with 50-99\% stenosis who have a TIA or stroke while on antithrombotic therapy (24). As such, use of Wingspan in the USA far exceeds that of any other stent (e.g. coronary stents) for treatment of intracranial stenosis. Moreover, the Wingspan delivery system was based on the self-expanding Neuroform stent system to treat intracranial aneurysms (both manufactured by Boston Scientific Corporation), which is a device that most neuro-interventionists have used extensively. Lastly, two post-marketing Wingspan registries led by the principal investigators of SAMMPRIS showed excellent technical success rates (96.7-98.8\%) and 30-day and longer stroke outcomes in patients with $70-99 \%(20,21)$ that appeared favorable compared to the outcomes in medically treated patients with 70-99\% stenosis in WASID (7).

\section{Why is combination antiplatelet therapy being used for 90 days in}

SAMMPRIS?-Aspirin and clopidogrel are used for a minimum of 30 days after PTAS, but the optimal duration of combination antiplatelet therapy is uncertain. The most common practice amongst interventionists who we surveyed when designing SAMMPRIS is to use aspirin and clopidogrel for 90 days after PTAS followed by aspirin alone. This, coupled with the results of MATCH (25) and CHARISMA (26) that suggest the risk of major hemorrhage on combination aspirin and clopidogrel increases after 90 days, provided the rationale for limiting the use of combination clopidogrel and aspirin for 90 days following PTAS in SAMMPRIS. We chose identical antiplatelet therapy for the medical arm for three main reasons: 1) to keep medical management in both arms the same so that any difference in outcome between the two arms of the study could be ascribed to PTAS alone; 2) MATCH 
and CHARISMA both suggested that combination antiplatelet therapy may be more effective than monotherapy in patients enrolled within 30 days of their qualifying event $(25,26)$, a requirement for enrollment in SAMMPRIS; and 3) the pathophysiology of recent TIA or stroke related to intracranial atherosclerotic stenosis is probably similar to the pathophysiology of acute coronary syndromes, which the CURE trial showed was more effectively treated with aspirin and clopidogrel than aspirin alone (27).

What is the rationale for the components of intensive risk factor management in SAMMPRIS?-Several factors suggested that intensive management of atherosclerotic risk factors should be incorporated into the design of SAMMPRIS: 1) WASID showed that poorly controlled blood pressure and elevated cholesterol were significantly associated with a higher rate of stroke, MI, or vascular death (28);2) other secondary prevention stroke trials have shown that treatment of elevated LDL (SPARCL trial) (29) and blood pressure (PROGRESS trial) (30) reduce the risk of recurrent stroke; 3) intensive medical management in patients with coronary artery disease had been shown to be as effective as endovascular intervention and usual medical management for preventing cardiac ischemic events (31) when SAMMPRIS was being designed (a subsequent trial showed that intensive medical management was as effective as intensive medical management plus endovascular treatment for stable $\mathrm{CAD}(32)$ ). We chose hypertension and raised LDL as the primary risk factors to target because WASID suggested that poor control of these two factors was associated with major vascular events (28). The target level of systolic blood pressure $<140$ $\mathrm{mm} \mathrm{Hg} \mathrm{(<130} \mathrm{mm} \mathrm{Hg} \mathrm{in} \mathrm{diabetics)} \mathrm{was} \mathrm{based} \mathrm{on} \mathrm{the} \mathrm{JNC7} \mathrm{recommendations} \mathrm{(33)} \mathrm{and} \mathrm{the}$ target for LDL $<70 \mathrm{mg} / \mathrm{dl}$ was based on the NCEP-ATPIII guidelines for very high-risk patients (16). Rosuvastatin was chosen for use in the trial because it is the most effective statin for LDL reduction on a milligram-to-milligram basis $(34,35)$ and the manufacturer of rosuvastatin generously agreed to provide it free of charge to study patients. Lastly, the lifestyle modification program was included because there is a large gap between recommended target levels for risk factors and those achieved in clinical practice (36-38) and lifestyle modification programs have been shown to reduce that gap (39-40).

\section{How does the design of SAMMPRIS reduce bias favoring one or the other} arm?-This was done by: 1) requiring identical aggressive medical management in both arms (with the exception of not providing a loading dose of clopidogrel in the medical arm); 2) not requiring the study neurologist to evaluate patients after PTAS. If study neurologists are required to see all patients after PTAS but do not examine patients in the medical arm at the same time shortly after enrollment (which is impractical since most are not hospitalized), that could lead to surveillance bias against the PTAS arm. Instead, we require the study coordinators to contact patients in both arms on day 4 after enrollment to determine if any adverse events have occurred; 3 ) defining ischemic stroke based on clinical criteria alone (i.e., not including DWI positive MRI lesions without persistent neurological signs at 24 hours as strokes) to minimize surveillance bias that would result from differential use of brain imaging in the two treatment arms (e.g., patients in the PTAS arm are more likely to undergo MRI in the peri-enrollment period because they are hospitalized for the PTAS procedure); 4) requiring a second neurologist, who is unaware of treatment assignment, to evaluate patients with events that may be difficult to classify as a stroke (prolonged TIAs, minor stroke); and 5) central adjudication of all potential endpoints by independent neurologists and cardiologists who are unaware of treatment assignment and date of enrollment so they cannot assume that early events are more likely to be in the PTAS arm.

\section{Unique Features of SAMMPRIS Design}

SAMMPRIS has several unique design features that may be worth incorporating into future secondary prevention trials in stroke. First, we are using aggressive protocol driven 
management of multiple vascular risk factors as part of the study protocol, with regular performance feedback and suggestions from the coordinating center to improve risk factor management at each site. Second, a lifestyle modification program is used to coach patients about healthy habits. Third, in deciding the hypothesized effect size for the benefit from PTAS, we used a national survey of physicians to quantify the minimum benefit that most physicians would want to see from PTAS to make it their treatment of choice. Historically, the hypothesized effect size for the benefit of one treatment over another in many trials has been based on estimating the treatment effect that physicians and patients would deem important enough to adopt a new treatment, as well as the feasibility of recruiting the required sample size with the estimated effect size. The use of physician surveys (and ideally patient surveys) may provide a more reliable approach for determining the clinically meaningful benefit that would lead to choosing one treatment over another. Finally, we established a contract with a national retail pharmacy chain to dispense study medications to patients wherever they live. This mimics clinical practice, is convenient to patients and study coordinators, maximizes efficiency (no need to mail medications if patients run out, no need to keep track of drug expirations), and lowers study costs (no need to have a drug distribution center ordering medications in bulk and supplying the sites, no wastage of medications, no need to monitor stocks at each site).

\title{
Acknowledgments
}

This study was funded by a research grant (U01 NS058728) from the US Public Health Service National Institute of Neurological Disorders and Stroke (NINDS).

\begin{abstract}
Corporate Support: Stryker Neurovascular (formerly Boston Scientific Neurovascular) provides study devices and supplemental funding for third party device distribution, site monitoring and study auditing. This research is also supported by the Investigator-Sponsored Study Program of AstraZeneca that donates rosuvastatin (Crestor) to study patients.
\end{abstract}

Vendors: Nationwide Better Health - INTERVENT provides the lifestyle modification program to the study at a discounted rate. The Regulatory and Clinical Research Institute (RCRI) (Minneapolis, MN) provided assistance in designing the site monitoring processes and perform the site monitoring visits. The VA Cooperative Studies Program Clinical Research Pharmacy Coordinating Center (Albuquerque, NM) handles the procurement, labeling, distribution, and inventory management of the study devices and rosuvastatin. Walgreens pharmacies provide study medications except rosuvastatin to patients at a discounted price (paid for by the study).

The PACE self-assessment forms for physical activity and smoking cessation were provided by the San Diego Center for Health Interventions, LLC.

We are indebted to: the patients who are participating in this study, Drs. Oscar Benavente, Carole White, Robert Hart, Pablo Pergola, and Ana Roldan from the SPS3 trial who assisted with the development and implementation of the SAMMPRIS blood pressure management protocol, Drs. George Howard and Tom Brott for providing advice on study design and other issues based on their experience with the CREST trial, and Rie Calcaterra who helped with the SAMMPRIS grant application and the launching of the trial.

\section{Appendix. Administrative Structure and Participants}

Executive Committee - M Chimowitz, C Derdeyn, D Fiorella, M Lynn, T Turan, B Lane, J Malloy, S Janis (NIH/NINDS member)

Site Representatives on Steering Committee* - H Lutsep, S Barnwell, M Waters, B Hoh, M Hourihane, E Levy, A Alexandrov, M Harrigan, D Chiu, R Klucznik, J Clark, C McDougall, M Johnson, L Pride, M Torbey, S Zaidat

Operations Committee - M Chimowitz, M Lynn, T Turan, B Lane, J Malloy, J Montgomery, S Swanson, J Cleveland, A Nizam 
Clinical Coordinating Center (CCC) - M Chimowitz, T Turan, B Lane, J Malloy,

Department of Neurosciences, Medical University of South Carolina, Charleston, SC.

Statistical Coordinating Center (SCC) - M Lynn, J Montgomery, A Nizam, G Cotsonis, S Swanson, J Cleveland, L Lu. Department of Biostatistics, Emory University, Rollins School of Public Health, Atlanta, GA.

Imaging Center - C Derdeyn, B Vendt, Mallinckdrodt Institute of Radiology, Washington University, St. Louis, MO

Central Neuroradiologists - H Cloft **, Z Rumboldt

Neurology Adjudication Committee - L Caplan, D Anderson, R Hart, R Bernstein

Cardiology Adjudication Committee - T O’Brien, G Hendrix, E Powers, S Chiaramida

Risk Factor Advisory Committee - T Turan, B Egan, M Lopes-Virella, K Hermayer, A Le, O Benavente, C White, M Chimowitz, W. Virgil Brown, B Lane

Interventional Credentialing Committee - C Derdeyn, D Fiorella, S Barnwell, J Dion, P Meyers, P Gobin, S Zaidat, B Lane (non-voting), M Chimowitz (non-voting)

Conflict of Interest Committee - M Williams, C Case, G Lanzino

National Institutes of Health/National Institute of Neurological Disorders and Stroke Liaison - C Moy, W Koroshetz

Safety Monitors - B Stern, G Zoarski

Data and Safety Management Board (DSMB) - J Grotta (Chair), D Hess, M Johnson, J Verter

Research Assistants - J Kandl, M Steiner, A Stufflebean, N Vilardo

Clinical Sites (highest enrolling sites listed first)

Oregon Health Sciences University - S Barnwell, H Lutsep, B Petersen, G Nesbit, W Clark, J Fields, A Har-el, D Larsen, S Enochs, K Feest, B Dugan, S Jamieson

University of Florida - B Hoh, M Waters, B Miller, A Khanna, T Sheehan, L Carlton, R Boyette, N Wilson

State University of New York at Buffalo - E Levy, A Siddiqui, M Hourihane, R Sawyer, C Ionita, M Donovan, A Crumlish, M Hartney

University of Alabama - M Harrigan, A Alexandrov, J Rothrock, I Lopez, D Patterson, L Cava, T Taylor, L Nelson, B Casey, K Albright

The Methodist Hospital Houston - R Klucznik, D Orlando, D Chiu, J Ling, J Volpi, J Ling, D McCane, L Katz

Barrow Neurological Institute- C McDougall, F Albuquerque, D Fiorella, J Clark, M Flaster, J Frey, G Monuel-Cobos, S Ladha, B Kasel, J Cacciola, S Porter, C Kelly, N Honea, H Jahnke 
The University of Texas Southwestern Medical Center at Dallas - L Pride, P Purdy, B Welch, M Johnson, J Lee, R Novakovic, C Hall, K Johnson

Medical College of Wisconsin - O Zaidat, M Torbey, D Book, J Lynch, B Fitzsimmons, A Helms, C Pierzchalski, J Delap, C Miller, E Brandenburg

Riverside Methodist Hospital, Columbus- R Budzik, P Pema, G Eubank, K Johnson, N Sample, M Taylor, W Metz, L Allison, T Campbell, K Tanner, A Garcia, A Bailey

Wayne State University - S Chaturvedi, A Xavier, K Rajamani, C Ranganathan, R Madhaven, F Mada, K Sawaya, L Palm, D Murray, D Vaphiadis

Medical University of South Carolina - A Turk, M Chimowitz, T Turan, I Chaudry, R Turner, R Adams, N Papamitsakis, A Walker, G Starr, E Debenham, J Garry, J Peterson

MultiCare / Tacoma General Hospital - B Kott, J Huddlestone, A Nohara, L Lyman, Y Zhu, M Barnhart, C Holsey

Moses Cone Health System - S Deveshwar, P Sethi, C Dohmeier, Y Yan, M Reynolds, K Willis, C Weymann, D Champey, W Hickling, W Harbison, D Parker, A Howard, S Hammonds, A Overton, T Goodpasture, P Henderson, C Martin

State University of New York at Stony Brook - H Woo, D Fiorella, C Perkins, L Donarummo, M Guido, L Krupp, P Coyle, O Gerber, S Fiore, D Madigan, M Baumeister, V Geronimo, D McHugh

Forsyth Medical Center - D Heck, C Chase, A Runheim, H Kraft, J Dodds, D Norwood, Y Whitley, T Browning, M Burdette

West Virginia University - A Rai, C Brooks, C Nance, L Gutmann, P Altemus, J Domico, M Power, T Weimer, R Whitescarver

Cleveland Clinic - P Rasmussen, T Masaryk, R Gupta, I Katzan, K Uchino, J Gebel, M Hussain, M Lu, C Bae, J Provencio, D Andrews-Hinders, T Wheeler, L Strozniak, N Tighe

Henry Ford Medical Center - M Kole, P Mitsias, A Katramados, A Russman, B Silver, N Sripathi, J Jones

Florida Hospital, Orlando - F Hellinger, D Honeycutt, L Bilanovic, B Brutsch, K Beaudry

University of Pittsburgh - T Jovin, V Reddy, L Weschler, B Jankowitz, S Zaidi, M Horowitz, R Lin, M Hammer, M Jumaa, S DeCesare, D Crowley-Lisowski, A Adams, J Oakley, J Billigen

St. Luke's Episcopal Hospital, Houston - M Mawad, H Morsi, E Bershad, J Suarez, G Lopez, S Moore, M Pierce, G DeFreitas

Columbia University Medical Center - P Meyers, M Elkind, J Willey, J Roberts, T Corporan University of Mississippi Medical Center - R Buciuc, H Uschmann, R Leacock, E Brumfield, M Bankston

Emory University - F Tong, F Nahab, J Dion, C Cawley, M Frankel, R Gupta, R Nogueria, A Webb, L Ayala, K Hanson, E Holland 
University of Miami - D Yavagal, J Romano, M Aziz-Sultan, M Katsnelson, S Koch, I Bustillo, M Lichtenberger

Weill Medical College of Cornell University - Y Gobin, D Leifer, H Riina, M Fink, D Jamieson, A Segal, H White, J Caronna, B Nikolov, K Salvaggio, M Velcheva, M Ogg

Rush University Medical Center- D Lopes, S Prabhakaran, V Lee, M Shanks, M Catalano, G Griffin, T Cole, C Woods

University of Pennsylvania - R Hurst, S Kasner, J Weigele, B Cucciara, S Messe, N Shafi, M Mullen, D Rose, I Rybinnik, L Sansing, M McGarvey, E Augelli, M DeSanto, N Gallatti, L Ventura, J Luciano

University of Cincinnati - T Abruzzo, A Ringer, P Khatri, D Kanter, M Flaherty, S Kempisty, K Sullivan, C Moore, L Sprafka, C Koenig, B Reinert

Central DuPage Hospital - H Shownkeen, A Mazumdar, H Echiverri, R Bajwa, K Dzamashvelli, R Sucholeiki, J Smith, S Lidtke, P Pastore

Case Western Reserve University - R Tarr, J Sunshine, C Sila, E Westbrook, M DeGeorgia, S Pundik, S Sundararajan, A Furlan, D Korosec, D Diorio

Cedars Sinai Medical Center - M Alexander, D Palestrant, P Lyden, C Miller, R Singh, C Serrano, E Ferguson, F Lin, P Kornbluth, T Zorge

Duke University Medical Center- T Smith, C Graffagnino, V Chilukuri, J Stoner, D Bombard

Erlanger Medical Center- B Baxter, D Rankine, T Devlin, D Jones, J Sparks, D Gaddis

Baylor Medical Center, Dallas - I Thacker, J Hise, D Graybeal, R Choudry, A Okai, E Broyles, M Johnson, R Smith, M Sams

Sacred Heart Medical Center - C Zylak, M Geraghty, T Powell, W Bender, E Rawner, R Franks, B Aaron, T Cantrell

Thomas Jefferson University - R Rosenwasser, P Jabbour, C Pineda, R Bell, D Brock, D August, E Simmons, A Salvatore, M Wakefield, M Sheridan, K Salgado

Scott \& White Memorial Hospital - W Lesley, R Lenehan, J Clark, R Wardlow, B Wulbrecht, M Carney, S Taggart

Glendale Adventist Medical Center - G Rappard, D Thompson, L Lee, C Galicia, J Cann, I Zoltzman, A Ghosalkar

Providence Medical Center, Southfield, MI - R Fessler, J Mick, B Kole, B Silverman, P Cullis, M Holtzman, N Warra, Y, Will- Murphy, M Silverman, K Telck, V Gordon, J Kelly, M Lahey, N Zakem, C Rieck

Carolinas Medical Center - J Bernard, S Dilbert, J Story, J Martin, M Price

Sentara Medical Center - J Agola, R Zweifler, J Rathbun, S Cummings

Massachusetts General Hospital - J Pryor, S Silverman, R Nogueira, F Buoanno, M Ning, A Singhal, N Rost, B Rista, A Chutinet 
Inova Fairfax Medical Center - R Pergolizzi, C Putman, J Cochran, R Lipsky, R Benson, J Rosecan, M Greenberg, K John, C Restak, M Khalil, B Wyllie

University of California San Francisco - R Higashida, M Nguyen- Huynh, J Tatum, S Poisson, J Scanlon, C Yancey, S Stason

Good Samaritan Hospital, San Jose - R Malek, H Sachdev, N Sacchdev, M Emami, U KellyTolley

Mayo Clinic Arizona - B Chong, B Demaerschalk, M Aguilar, D Dodick, T Ingall, B Vargas, K Noe, J Sirven, J Drazkowski, E Boyd, J Wieser

Johns Hopkins University - P Gailloud, R Wityk, P Nyquist, B Kohler, A Jones

Washington University, St Louis - C Moran, C Derdeyn, D Cross, D Carpenter, A Ford, J Lee, A Zazulia, N Hantler, J Newgent, J Rickmann, J Serna, L Carpenter

Washington Hospital, Washington, DC - W Bank, A Liu, A Hsia, M Lin, M Schlosberg, J Martin, M Cotabato, I Fleming

* neurologists and interventionists from the top 8 enrolling sites

** on Steering Committee

\section{References}

1. Gorelick P, Wong K, Bae H, Pandey D. Large artery intracranial occlusive disease, A large worldwide burden but a relatively neglected frontier. Stroke. 2008; 39:2396-2399. [PubMed: 18535283]

2. Arenillas JF. Intracranial atherosclerosis: current concepts. Stroke. 2011; 42(1 Suppl):S20-S23. Epub 2010 Dec 16. Review. PubMed PMID: 21164126. [PubMed: 21164126]

3. Wong LKS. Global burden of intracranial atherosclerosis. Int Journal of Stroke. 2006; 1:158-159.

4. Wityk R, Lehman D, Klag M, et al. Race and sex differences in the distribution of cerebral atherosclerosis. Stroke. 1996; 27:1974-1980. [PubMed: 8898801]

5. Sacco RL, Kargman D, Gu Q, Zamanillo MC. Race-ethnicity and determinants of intracranial atherosclerotic cerebral infarction. The Northern Manhattan Stroke Study. Stroke. 1995; 26:14-20. [PubMed: 7839388]

6. Chimowitz MI, Lynn MJ, Howlett-Smith H, et al. Warfarin-Aspirin Symptomatic Intracranial Disease Trial Investigators. Comparison of warfarin and aspirin for symptomatic intracranial arterial stenosis. N Engl J Med. 2005; 352:1305-1316. [PubMed: 15800226]

7. Kasner S, Chimowitz M, Lynn M, et al. Warfarin Aspirin Symptomatic Intracranial Disease Trial Investigators. Predictors of ischemic stroke in the territory of a symptomatic intracranial arterial stenosis. Circulation. 2006; 113:555-563. [PubMed: 16432056]

8. Fiorella DF, Turan TN, Derdeyn CP, Chimowitz MI. Current status of the management of symptomatic intracranial atherosclerotic disease: The rationale for a randomized trial of medical therapy and intracranial stenting. Journal of NeuroInterventional Surgery. 2009; 1:35-39. [PubMed: 21994103]

9. Rorick MB, Nichols FT, Adams RJ. Transcranial Doppler correlation with angiography in detection of intracranial stenosis. Stroke. 1994; 25:1931-1934. [PubMed: 7916499]

10. Feldmann E, Wilterdink JL, Kosinski A, et al. The Stroke Outcomes and Neuroimaging of Intracranial Atherosclerosis (SONIA) trial. Neurology. 2007; 68:2099-2106. [PubMed: 17409371]

11. Katz DA, Marks MP, Napel SA, et al. Circle of Willis: evaluation with spiral CT angiography, MR angiography, and conventional angiography. Radiology. 1995; 195:445-449. [PubMed: 7724764]

12. Nguyen-Huynh MN, Wintermark M, English J, et al. How accurate is CT angiography in evaluating intracranial atherosclerotic disease? Stroke. 2008; 39:1184-1188. [PubMed: 18292376] 
13. Samuels OB, Joseph GJ, Lynn MJ, et al. A standardized method for measuring intracranial arterial stenosis. American Journal of Neuroradiology. 2000; 21:643-646. [PubMed: 10782772]

14. Meyers PM, Higashida RT, Phatouros CC, et al. Cerebral hyperperfusion syndrome after percutaneous transluminal stenting of the craniocervical arteries. Neurosurgery. 2000; 47(2):335343. [PubMed: 10942006]

15. American Diabetes Association. Standards of medical care in diabetes--2006. Diabetes Care. 2006; 29(Suppl 1):S4-S42. [PubMed: 16373931]

16. Grundy SM, Cleeman JI, Merz CNB, et al. for the coordinating Committee of the National Cholesterol Education Program (Endorsed by the National Heart, Lung, and Blood Institute, American College of Cardiology Foundation, and American Heart Association). Implications of recent clinical trials for the National Cholesterol Education Program Adult Treatment Panel III guidelines. Circulation. 2004; 110:227-239. [PubMed: 15249516]

17. Fiore, MC.; Bailey, WC.; Cohen, SJ., et al. Quick Reference Guide for Clinicians. Rockville, MD: U.S. Department of Health and Human Services. Public Health Service; 2000 Oct. Treating Tobacco Use and Dependence.

18. Poirier P, Giles TD, Bray GA, et al. American Heart Association. Obesity Committee of the Council on Nutrition, Physical Activity, and Metabolism. Obesity and cardiovascular disease: pathophysiology, evaluation, and effect of weight loss: an update of the 1997 American Heart Association Scientific Statement on Obesity and Heart Disease from the Obesity Committee of the Council on Nutrition, Physical Activity, and Metabolism. Circulation. 2006; 113(6):898-918. [PubMed: 16380542]

19. Turan TN, Lynn M, Chimowitz MI. Survey of US Stroke Neurologists and Neurointerventionalists on Treatment Choices for Intracranial Stenosis. Cerebrovascular Diseases. 2007; 23(S2):131. (abstract).

20. Fiorella D, Levy EI, Turk AS, et al. US multicenter experience with the wingspan stent system for the treatment of intracranial atheromatous disease: periprocedural results. Stroke. $2007 ; 38: 881-$ 887. [PubMed: 17290030]

21. Zaidat OO, Klucznik R, Alexander MJ, et al. NIH Multi-center Wingspan Intracranial Stent Registry Study Group. The NIH registry on use of the Wingspan stent for symptomatic 70-99\% intracranial arterial stenosis. Neurology. 2008; 70:1518-1524. [PubMed: 18235078]

22. O’Brien PC, Fleming TR. A multiple testing procedure for clinical trials. Biometrics. 1979; 35:549-556. [PubMed: 497341]

23. Jiang WJ, Xu XT, Du B, et al. Comparison of elective stenting of severe vs moderate intracranial atherosclerotic stenosis. Neurology. 2007; 68:420-426. [PubMed: 17283316]

24. Bose A, Hartmann M, Henkes H, et al. A novel, self-expanding, nitinol stent in medically refractory intracranial atherosclerotic stenoses: the Wingspan study. Stroke. 2007; 38(5):15311537. Epub 2007 Mar 29. PubMed PMID: 17395864. [PubMed: 17395864]

25. Diener HC, Bogousslavsky J, Brass LM, et al. MATCH investigators. Aspirin and clopidogrel compared with clopidogrel alone after recent ischaemic stroke or transient ischaemic attack in high-risk patients (MATCH): randomised, double-blind, placebo- controlled trial. Lancet. 2004; 364(9431):331-337. [PubMed: 15276392]

26. Bhatt DL, Fox KAA, Hacke W, et al. for the CHARISMA investigators. Clopidogrel and aspirin versus aspirin alone for the prevention of atherothrombotic events. N Engl J Med. 2006; 354:1706-1717. [PubMed: 16531616]

27. The Clopidogrel in Unstable Angina to Prevent Recurrent Events Trial Investigators. Effects of clopidogrel in addition to aspirin in patients with acute coronary syndromes without ST-segment elevation. N Engl J Med. 2001; 345:494-502. [PubMed: 11519503]

28. Chaturvedi S, Turan TN, Lynn MJ, et al. for the WASID Study Group. Risk factor status and subsequent vascular events in patients with symptomatic intracranial stenosis. Neurology. 2007; 69:2063-2068. [PubMed: 18040012]

29. The Stroke Prevention by Aggressive Reduction in Cholesterol Levels (SPARCL) Investigators. High-dose atorvastatin after stroke or transient ischemic attack. N Engl J Med. 2006; 355:549_ 559. [PubMed: 16899775] 
30. PROGRESS Collaborative Group. Randomised trial of a perindopril-based blood-pressurelowering regimen among 6105 individuals with previous stroke or transient ischaemic attack. Lancet. 2001; 358:1033-1041. [PubMed: 11589932]

31. Pitt B, Waters D, Brown WV, et al. Aggressive lipid-lowering therapy compared with angioplasty in stable coronary artery disease. Atorvastatin versus Revascularization Treatment Investigators. New England Journal of Medicine. 1999; 341(2):70-76. [PubMed: 10395630]

32. Boden WE, O'Rourke RA, Teo KK, et al. COURAGE Trial Research Group. Optimal medical therapy with or without PCI for stable coronary disease. N Engl J Med. 2007; 356(15):1503-1506. Epub 2007 Mar 26. PubMed PMID: 17387127. [PubMed: 17387127]

33. Chobanian AV, Bakris GL, Black HR, et al. National Heart, Lung, and Blood Institute Joint National Committee on Prevention, Detection, Evaluation, and Treatment of High Blood Pressure; National High Blood Pressure Education Program Coordinating Committee. The seventh report of the Joint National Committee on Prevention, Detection, Evaluation, and Treatment of High Blood Pressure: the JNC 7 report. JAMA. 2003; 289:2560-2572. [PubMed: 12748199]

34. Brown WV, Bays HE, Hassman HA, et al. Efficacy and safety of rosuvastatin compared with pravastatin and simvastatin in patients with hypercholesterolemia: a randomised, double-blind, 52week trial. Am Heart J. 2002; 144:1036-1043. [PubMed: 12486428]

35. Jones PH, Davidson MH, Stein EA, et al. STELLAR Study Group. Comparison of the efficacy and safety of rosuvastatin versus atorvastatin, simvastatin, and pravastatin across doses (STELLAR* Trial). American Journal of Cardiology. 2003; 92(2):152-160. [PubMed: 12860216]

36. Pearson TA, Peters TD. The treatment gap in coronary artery disease and heart failure; community standards and the post-discharge patient. Am J Cardiol. 1997; 80(suppl 8B):45H-52H. [PubMed: 9205018]

37. Saposnik G, Goodman SG, Leiter LA, et al. Applying the evidence: do patients with stroke, coronary artery disease, or both achieve similar treatment goals? Stroke. 2009; 40(4):1417-1424. [PubMed: 19213947]

38. Sanossian N, Ovbiagele B. Multimodality Stroke Prevention. The Neurologist. 2006; 12(1):14-31. [PubMed: 16547443]

39. Gordon NF, Salmon RD, Franklin BA, et al. Effectiveness of therapeutic lifestyle changes in patients with hypertension, hyperlipidemia, and / or hyperglycemia. Am J Cardiol. 2004; 94:15581561. [PubMed: 15589017]

40. Gordon NF, English CD, Contractor AS, et al. Effectiveness of three models for comprehensive cardiovascular disease risk reduction. Am J Cardiol. 2002; 89(11):1263-1268. [PubMed: 12031725] 


\section{Table 1}

\section{INCLUSION CRITERIA}

1 TIA or non-severe stroke within 30 days of enrollment attributed to 70-99\% stenosis * of a major intracranial artery (carotid artery, MCA stem (M1), vertebral artery, or basilar artery)

* may be diagnosed by TCD, MRA, or CTA to qualify for angiogram performed as part of the study protocol but must be confirmed by catheter angiography for enrollment in the trial

$2 \quad$ Modified Rankin score of $\leq 3$

3 Target area of stenosis in an intracranial artery that has a normal diameter of $2.00 \mathrm{~mm}$ to $4.50 \mathrm{~mm}$

4 Target area of stenosis is less than or equal to $14 \mathrm{~mm}$ in length

$5 \quad$ Age $\geq 30$ years and $\leq 80$ years.

- Patients 30-49 years are required to meet at least one additional criteria (i-vi) provided in the table below to qualify for the study. This additional requirement is to increase the likelihood that the symptomatic intracranial stenosis in patients $30-49$ years is atherosclerotic.

i. insulin dependent diabetes for at least 15 years

ii. at least 2 of the following atherosclerotic risk factors: hypertension (BP $\geq 140 / 90$ or on antihypertensive therapy); dyslipidemia (LDL $\geq 130 \mathrm{mg} / \mathrm{dl}$ or HDL $<40 \mathrm{mg} / \mathrm{dl}$ or fasting triglycerides $\geq 150 \mathrm{mg} / \mathrm{dl}$ or on lipid lowering therapy); smoking; non-insulin dependent diabetes or insulin dependent diabetes of less than 15 years duration; family history of any of the following: myocardial infarction, coronary artery bypass, coronary angioplasty or stenting, stroke, carotid endarterectomy or stenting,peripheral vascular surgery in parent or sibling who was $<55$ years of age for men or $<65$ for women at the time of the event

iii. history of any of the following: myocardial infarction, coronary artery bypass, coronary angioplasty or stenting, carotid endarterectomy or stenting, or peripheral vascular surgery for atherosclerotic disease

iv. any stenosis of an extracranial carotid or vertebral artery, another intracranial artery, subclavian artery,coronary artery, iliac or femoral artery, other lower or upper extremity artery, mesenteric artery, or renal artery that was documented by non-invasive vascular imaging or catheter angiography and is considered atherosclerotic

v. aortic arch atheroma documented by non-invasive vascular imaging or catheter angiography

vi. any aortic aneurysm documented by non-invasive vascular imaging or catheter angiography that is considered atherosclerotic

$6 \quad$ Negative pregnancy test in a female who has had any menses in the last 18 months

$7 \quad$ Patient is willing and able to return for all follow-up visits required by the protocol

8 Patient is available by phone

9 Patient understands the purpose and requirements of the study, can make him/herself understood, and has provided informed consent 
Table 2

\section{EXCLUSION CRITERIA}

1. Tandem extracranial or intracranial stenosis (70\%-99\%) or occlusion that is proximal or distal to the target intracranial lesion (NOTE: an exception is allowed if the occlusion involves a single vertebral artery proximal to a symptomatic basilar artery stenosis and the contralateral vertebral artery is supplying the basilar artery)

2. Bilateral intracranial vertebral artery stenosis of $70 \%-99 \%$ and uncertainty about which artery is symptomatic (e.g. if patient has pontine, midbrain, or temporal - occipital symptoms)

3. Stenting, angioplasty, or endarterectomy of an extracranial (carotid or vertebral artery) or intracranial artery within 30 days prior to expected enrollment date

4. Previous treatment of target lesion with a stent, angioplasty, or other mechanical device, or plan to perform staged angioplasty followed by stenting of target lesion

5. Plan to perform concomitant angioplasty or stenting of an extracranial vessel tandem to an intracranial stenosis

6. Presence of intraluminal thrombus proximal to or at the target lesion

7. Any aneurysm proximal to or distal to stenotic intracranial artery

8. Intracranial tumor (except meningioma) or any intracranial vascular malformation

9. CT or angiographic evidence of severe calcification at target lesion

10. Thrombolytic therapy within 24 hours prior to enrollment

11. Progressive neurological signs within 24 hours prior to enrollment

12. Brain infarct within previous 30 days of enrollment that is of sufficient size $(>5 \mathrm{cms})$ to be at risk of hemorrhagic conversion during or after stenting

13. Any hemorrhagic infarct within 14 days prior to enrollment

14. Any hemorrhagic infarct within 15 - 30 days that is associated with mass effect

15. Any history of a primary intracerebral (parenchymal) hemorrhage (ICH)

16. Any other intracranial hemorrhage (subarachnoid, subdural, epidural) within 30 days

17. Any untreated chronic subdural hematoma of greater than $5 \mathrm{~mm}$ in thickness

18. Intracranial arterial stenosis due to arterial dissection, Moya Moya disease; any known vasculitic disease; herpes zoster, varicella zoster or other viral vasculopathy; neurosyphilis; any other intracranial infection; any intracranial stenosis associated with CSF pleocytosis; radiation induced vasculopathy; fibromuscular dysplasia; sickle cell disease; neurofibromatosis; benign angiopathy of central nervous system; post-partum angiopathy; suspected vasospastic process, suspected recanalized embolus

19. Presence of any of the following unequivocal cardiac sources of embolism: chronic or paroxysmal atrial fibrillation, mitral stenosis, mechanical valve, endocarditis, intracardiac clot or vegetation, myocardial infarction within three months, dilated cardiomyopathy, left atrial spontaneous echo contrast, ejection fraction less than $30 \%$

20. Known allergy or contraindication to aspirin, clopidogrel, heparin, nitinol, local or general anesthesia

21. History of life-threatening allergy to contrast dye. If not life threatening and can be effectively pretreated, patient can be enrolled at physician's discretion

22. Active peptic ulcer disease, major systemic hemorrhage within 30 days, active bleeding diathesis, platelets $<100,000$, hematocrit $<30$, INR $>1.5$, clotting factor abnormality that increases the risk of bleeding, current alcohol or substance abuse, uncontrolled severe hypertension (systolic pressure $>180 \mathrm{~mm} \mathrm{Hg}$ or diastolic pressure $>115 \mathrm{~mm} \mathrm{Hg}$ ), severe liver impairment (AST or ALT $>3 \mathrm{x}$ normal, cirrhosis), creatinine $>3.0$ (unless on dialysis)

23. Major surgery (including open femoral, aortic, or carotid surgery) within previous 30 days or planned in the next 90 days after enrollment

24. Indication for warfarin or heparin beyond enrollment (NOTE: exceptions allowed for use of systemic heparin during stenting procedure or subcutaneous heparin for deep vein thrombosis (DVT) prophylaxis while hospitalized)

25. Severe neurological deficit that renders the patient incapable of living independently

26. Dementia or psychiatric problem that prevents the patient from following an outpatient program reliably

27. Co-morbid conditions that may limit survival to less than 3 years

28. Pregnancy or of childbearing potential and unwilling to use contraception for the duration of this study

29. Enrollment in another study that would conflict with the current study. 